\title{
ANÁLISE ESTATÍSTICA ENTRE OS DADOS DE PRECIPITAÇÃO ESTIMADA VIA SATÉLITE TRMM E DADOS OBSERVADOS DE SUPERFÍCIE EM PRAIA - CABO VERDE
}

\author{
ALVES, Keylyane Santos da Silva - keylyanesds@gmail.com \\ Universidade Federal de Mato Grosso/UFMT \\ ALVES, Alan Vitor Pinheiro - vpalvesalan@gmail.com \\ Universidade Federal de Mato Grosso/UFMT \\ SOUZA, Natallia Sanches e - natalliass@hotmail.com \\ Universidade Federal de Mato Grosso/UFMT \\ SANCHES, Luciana - Isanches@hotmail.com \\ Universidade Federal de Mato Grosso/UFMT \\ GAIO, Denilton Carlos - dcgaio@ufmt.br \\ Universidade Federal de Mato Grosso/UFMT \\ SOUZA, Gracyeli Santos - gracyeli@gmail.com \\ Universidade Federal de Mato Grosso/UFMT \\ DE MUSIS, Carlo Ralph - carlo.demusis@gmail.com \\ Universidade de Cuiabá/UNIC
}

\begin{abstract}
RESUMO: O arquipélago de Cabo Verde é formado por dez ilhas vulcânicas localizadas a $620 \mathrm{~km}$ da costa da África, no Oceano Atlântico. Neste arquipélago, as características espaciais e temporais das chuvas são influenciadas principalmente pela circulação atmosférica e podem ser entendidas por séries temporais de precipitação. Atualmente, os produtos de precipitação por satélite fornecem cada vez mais um meio alternativo para a rede de pluviometria de baixa densidade. Assim, o presente trabalho tem como objetivo comparar o produto de precipitação TRMM (Tropical Rainfall Measurement Mission) com dados pluviométricos de calibração sobre a cidade de Praia, Cabo Verde. Os produtos de precipitação TRMM utilizados foram 3B43 TRMM considerando várias escalas temporais e espaciais de 1998 a 2002. Os resultados indicaram uma relação significativa entre os dados mensais da precipitação TRMM e os dados dos pluviômetros. A precisão e a acurácia dos resultados das chuvas em Cabo Verde foram melhores que outras regiões tropicais. Concluímos que o produto TRMM é uma alternativa a ser utilizada para chuvas tropicais em regiões onde os pluviômetros são escassos e tem um grande potencial em aplicações do mundo real.
\end{abstract}

Palavras- chaves: Precipitação tropical, postos pluviométricos, sensoriamento remoto.

STATISTICAL ANALYSIS BETWEEN ESTIMATED PRECIPITATION DATA FROM TRMM SATELLITE AND OBSERVED SURFACE DATA ON BEACH - CAPE VERDE

ABSTRACT: The Cape Verde archipelago is formed by ten volcanic islands located 620 $\mathrm{km}$ off the coast of Africa, in the Atlantic Ocean. In this archipelago, the spatial and temporal characteristics of the rainfall is influenced mainly by the atmospheric circulation and can be understood by rainfall time series. Currently, satellite-based rainfall products increasingly provide an alternative way to low-density rain gauge network. Thus, the present work is aimed at comparing the TRMM (Tropical Rainfall Measurement Mission) rainfall product versus gauge rainfall data over city of Praia, Cape Verde. The TRMM rainfall products used was 3B43 TRMM considering several temporal and spatial scales 
from 1998 to 2002. The results indicated a significant relationship between TRMM monthly rainfall data and rain gauges data. The precision and accuracy of the results of rainfall over Cape Verde were better than another tropical region. We conclude that the TRMM product is an alternative to be used to tropical rainfall over regions where rain gauges are sparse and it has great potential in real-world applications.

Keywords: tropical rainfall, rain gauge, remote sensing.

\section{INTRODUÇÃO}

A compreensão e melhorias na qualidade da estimativa de precipitação pluviométrica atualmente tem grande importância, principalmente nos países em desenvolvimento, uma vez que ao analisar as variações espaço-temporais da precipitação em séries de dados de longa duração possibilita melhores previsões de chuva, contribuindo para o desenvolvimento social, econômico adequado e sustentável de uma região (HOU et al., 2011), por meio de modelos hidrológicos que apresentem simulações bem sucedidas (KITTEL et al., 2017).

A precipitação pluviométrica é a variável mais importante no ciclo hidrológico, possuindo grande influência na circulação atmosférica, pois, parte da energia gerada de tal circulação provem do calor latente, que é originado da precipitação tropical (KIDD e HUFFMAN, 2011). Os padrões de precipitações nos trópicos variam entre secas severas e cheias ocasionais, no entanto as chuvas duram cerca de horas por evento ocorrido, surgindo a necessidade de se instalar postos pluviométricos convencionais em superfície, já que estes são capazes de registrar com precisão tais eventos (COLLISCHONN, 2006).

Os postos pluviométricos estão suscetíveis a uma gama de problemas, dentre esses problemas pode-se ressaltar o processo de leitura dos dados realizada por um observador podendo ocorrer falhas. Outro fator de suma importância é que os postos pluviométricos só registram os dados de precipitação que ocorrem em sua escala de abrangência, portanto não registram precipitações quando estas ocorrem a uma maior distância, consequentemente, necessitando de uma quantidade de postos pluviométricos consideravelmente suficientes para suprir a demanda em uma dada região.

Porém, devido a problemas como falta de investimento por parte dos órgãos competentes, nem sempre há postos pluviométricos instalados suficientes em toda superfície ou em grande parte dela, além da dificuldade de acesso a regiões montanhosas e oceânicas, que ainda é um desafio para ciência, mas que tem grande importância quando se estuda o ciclo hidrológico (SILVA et al., 2013; NASTOS et al., 2016).

A estimativa de precipitação pluviométrica usando o sensoriamento remoto contribui para o aumento de cobertura de dados climatológicos. O uso dessa técnica se tornou indispensável para suprir a escassez de dados em regiões que não possuem postos pluviométricos convencionais ou que não abrange todo o seu território (SANCHEZ-MORENO et al., 2014).

Para esse tipo de estudo, o satélite Tropical Rainfall Measuring Mission, Missão De Medição Das Chuvas Tropicais (TRMM) foi o mais utilizado, sendo um satélite concebido para medir estrutura vertical da precipitação nos trópicos (KUMMEROW et al., 1998; KUMMEROW et al., 2001; HUFFMAN et al., 2007; YANG e SMITH, 2008; MEHTA e YANG, 2008). Durante mais de 16 anos 
renderam centenas de grandes sistemas de precipitação ( $\geq 100 \mathrm{~km}$ ) para cada grade de $0,1^{\circ}$ nas principais regiões chuvosas (HIROSE et al., 2017).

Em 1997, a NASA (Administração Nacional de Aeronáutica e Espaço) em parceria com a JAXA (Agência Japonesa de Exploração Aeroespacial) lançava o TRMM com o objetivo específico de realizar medições de precipitação nos trópicos (SILVA et al., 2013).

O satélite possui órbita oblíqua não-heliossíncrona baixa (cerca de 403 $\mathrm{km}$ ), de forma que o período de translação é bastante curto (91 minutos) permitindo resoluções espacial e temporal comparativamente altos. Sua orbita foi calculada para se ter uma capacidade máxima de amostragem diurna e os dados são estimados a cada 3 horas com uma resolução espacial de $0,25^{\circ}$ desde $50^{\circ} \mathrm{N}$ a $50^{\circ} \mathrm{S}$. Os instrumentos a bordo do TRMM são: imageador de microondas (TMI), radar de precipitação (PR), radiômetro no visível e no infravermelho (VIRS), sensor de energia radiante da superfície terrestre e das nuvens (CERES), e sensor para imageamento de relâmpagos (LIS) (NASTOS et al., 2016).

A maioria das chuvas em Cabo Verde tem suas origens nas depressões tropicais que se desenvolvem sobre a África Ocidental e o golfo da Guiné e, portanto, são fortemente influenciadas pelas massas de ar tropical úmidas continentais provenientes da África. A ilha de Santiago é uma ilha semi-árida sob o regime climático do Sahel, apresenta um regime de precipitação sazonal caracterizado por estações secas (novembro-junho) e úmidas (julho-outubro), com curto período de transição (SANCHEZ-MORENO et al., 2014).

O relevo e as variações de elevação na Ilha de Santiago sugerem uma forte influência da topografia sobre a variabilidade pluviométrica, no entanto, não é possível caracterizá-la devido à forte variabilidade das chuvas associadas a escassez de dados sobre a ilha. Neste caso o sensoriamento remoto pode ser uma ferramenta útil para obtenção de dados.

Neste contexto, o objetivo deste estudo foi comparar a precipitação pluviométrica estimada pelo satélite TRMM com dados observados em postos pluviométricos instalados em superfície sobre a região de Praia Capital de Cabo Verde, durante os anos 1998 a 2002.

\section{MATERIAL E MÉTODOS}

\section{LOCALIZAÇÃO E DESCRIÇÃO DA ÁREA}

O arquipélago de Cabo Verde é um conjunto de dez ilhas situadas no atlântico norte, $\left(14^{\circ} 42 \mathrm{~N}\right)$ cerca de $600 \mathrm{Km}$ da costa ocidental do continente africano. As ilhas do arquipélago estão agrupadas em dois conjuntos definidos pela sua posição em relação aos ventos predominantes. O de Barlavento (Santo Antão, São Vicente, Santa Luzia, S. Nicolau, Sal, Boa Vista e os ilhéus de Branco e Raso) e o de Sotavento (Maio, Santiago, Fogo, Brava e os ilhéus Secos ou do Rombo) (SANCHEZ-MORENO et al., 2014).

O clima é tropical seco (região sub-saheliana), mas as temperaturas são relativamente amenas, devido à influência dos ventos alísios. A temperatura varia entre $30^{\circ} \mathrm{C}$ e $15^{\circ} \mathrm{C}$ (INMG). A pluviosidade é irregular, muito embora se distingam em duas estações: "as águas" e "as secas". O período das secas é 
muito longo e com pluviosidade praticamente nula. A estação "das águas" é o período que vai de agosto a outubro onde ocorrem chuvas irregulares de curta duração, consequentemente muito intensas e devastadoras, causadoras de inundações, enchentes e outros transtornos à população insular (COSTA, 1996).

Santiago é a maior ilha de Cabo Verde e pertence ao grupo do Sotavento, localizada entre os paralelos $15^{\circ} 20^{\prime} \mathrm{N}$ e $14^{\circ} 50^{\prime} \mathrm{N}$ e os meridianos $23^{\circ} 50^{\prime} \mathrm{O}$ e $23^{\circ} 20^{\prime} \mathrm{O}$. Estende-se por cerca de $75 \mathrm{~km}$ de comprimento, no sentido Norte-Sul e cerca de $35 \mathrm{Km}$ de largura, no sentido Leste-Oeste, contabilizando uma área total de aproximadamente $992 \mathrm{~km}^{2}$. Encontra-se dividida em 9 concelhos: Tarrafal, São Miguel, Santa Catarina, Santa Cruz, São Salvador do Mundo, São Lourenço dos Órgãos, São Domingos, Ribeira Grande e Praia (LIMA, 2012).

Assim como as outras ilhas Santiago é de origem vulcânica, ocorrendo a alternância de materiais piroclásticos e assentadas de basaltos. É uma ilha com maciços montanhosos com sua cobertura vegetal pouco densa.

De acordo com Lima (2012) as chuvas registradas no interior da ilha são muito intensas e rapidamente formam grandes quantidades de escoamento superficial que, em virtude do favorecimento da declividade, escoa no sentido da cidade da Praia localizada no litoral Sul da ilha, alagando áreas residenciais e vias públicas. Segundo dados do INE, Praia atingia em 2010 um total de 127832 habitantes, ou seja, $26 \%$ do total do país.

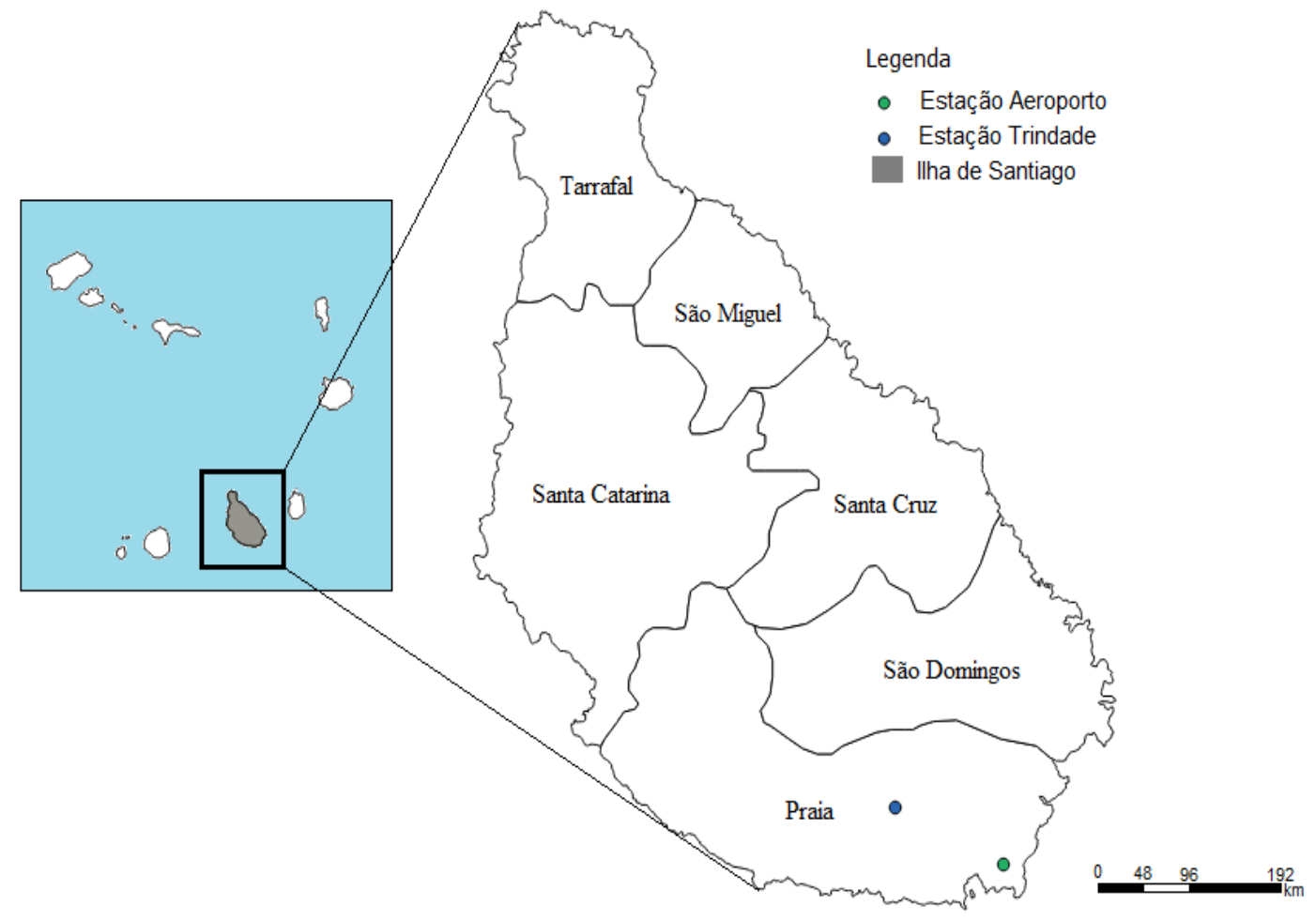

Figura 1 - Localização de Ilha de Santiago e dos postos pluviométricos instalados na cidade de Praia - Cabo Verde. 


\section{PRECIPITAÇÃO PLUVIOMÉTRICA ESTIMADA POR SENSORIAMENTO REMOTO}

Os dados de precipitação pluviométrica obtidos pelo satélite TRMM foram disponibilizados gratuitamente no site da NASA (www.nasa.gov), no período entre janeiro de 1998 a dezembro de 2002 . O produto 3B43 TRMM foi escolhido para realização do estudo por ser o produto de estimativa mensal para serem comparados com os dados observados de precipitação em postos pluviométricos em acumulados mensais.

O produto V5 3B43 TRMM é produzido pela fusão do acúmulo mensal diário do produto V5 3B42 TRMM com o acumulado mensal do Sistema de Monitoramento e Avaliação do Clima (CAMS) ou Centro Global de Climatologia e precipitação (GPCC) medidor de análise de chuva (3A45 TRMM) (ISLAM e UYEDA, 2007; HUFFMAN et al., 2015).

\section{$\begin{array}{lllll}\text { PRECIPITAÇÃO PLUVIOMÉTRICA OBTIDA } & \text { POR POSTOS }\end{array}$ PLUVIOMÉTRICOS}

Os dados de precipitação pluviométricas de superfície foram obtidos por postos pluviométricos instalados em Praia nas coordenadas $23^{\circ} 55^{\prime} \mathrm{N}$ e $23^{\circ} 29^{\prime}$ $\mathrm{O}$ à $64 \mathrm{~m}$ de altitude e Trindade nas coordenadas $1^{\circ} 57^{\prime} \mathrm{N}$ e $23^{\circ} 33^{\prime} \mathrm{O}$ à $204 \mathrm{~m}$ de altitude, ambas no mesmo período, disponibilizados pelo Instituto Nacional de Meteorologia e Geofísica (INMG).

\section{TRATAMENTO E ANÁLISE DOS DADOS}

Alguns parâmetros estatísticos foram determinados para avaliar a qualidade das estimativas obtidas pelo pixel específico que sobrepõe os postos pluviométricos. Portanto, verificar se as estimativas detectam corretamente 0 regime de precipitação anual de acordo com o que os postos pluviométricos registraram no período de estudo.

Assim, é necessário ressaltar que os índices aplicados aos dados foram de duas formas: (1) analisar os dados anualmente, ou seja, para cada ano aplicou-se todos os índices e (2) analisar a série temporal total.

O pixel utilizado no estudo coincidiu com os dois postos pluviométricos, portanto houve a necessidade de analisar os dados separadamente, cada posto pluviométrico foi analisado isoladamente com o pixel e posteriormente fez-se uma média entre os dados observados para analisá-los com os dados estimados pelo TRMM.

Foram utilizados índices específicos: coeficiente de correlação linear de Pearson ( $r$ ) equação (1), que indica a intensidade da associação linear existente entre as variáveis (medidas e estimadas), a raiz quadrada do erro médio quadrático (RMSE) equação (2), que indica o quanto de erro os dados estão sendo estimados pelo satélite em relação aos dados observados, e o erro relativo (Errv (\%)) equação (3) que nesse caso indica se o TRMM superestima ou subestima a precipitação. 


$$
\begin{array}{ll}
r=\frac{n \sum_{i=1}^{n} x_{i} y_{i}-\sum_{i=1}^{n} x_{i} \sum_{i=1}^{n} y_{i}}{\sqrt{\left[n \sum_{i=1}^{n} x_{i}^{2}-\left(\sum_{i=1}^{n} x_{i}\right)^{2}\right]\left[n \sum_{i=1}^{n} y_{i}^{2}-\left(\sum_{i=1}^{n} y_{i}\right)^{2}\right]}} & \text { Equação (1) } \\
R M S E=\sqrt{\frac{1}{n} \sum_{I=1}^{N}\left(x_{i}-y_{i}\right)^{2}} & \text { Equação (2) } \\
\operatorname{Errv}(\%)=\frac{\sum_{i=1}^{n}\left(x_{i}-y_{i}\right)}{\sum_{i=1}^{n} y_{i}} & \text { Equação (3) }
\end{array}
$$

Em que, $x$ é a precipitação estimada pelo produto TRMM e y é a precipitação medida nos postos pluviométricos.

Para a verificação quanto à eficácia do satélite em registrar os meses em que foram observados precipitação foram selecionados três índices categóricos:

- Probabilidade de Detecção (POD) (conforme equação (4)), que indica a razão entre o número de meses com precipitação detectados pelo satélite e o número de meses com precipitações observadas a partir dos postos pluviométricos. Se o satélite detectar corretamente os meses em que houve precipitação observada pelos pluviômetros o resultado será igual a 1 (um).

- Alarme Falso (FAR) (conforme equação (5)), que mensura a fração de precipitação que falsamente detectada pelo satélite, quando o resultado é igual a 0 (zero), indica que todas as precipitações detectadas pelo satélite foram observadas pelos pluviômetros, e quando o resultado é igual a 1 (um), as deteç̧ões são falsas, ou seja, nos meses em que o satélite detectou precipitação não houve registro nos postos pluviométricos.

- Índice de Sucesso (CSI) (conforme equação (6)), que indica a fração total de eventos que são corretamente detectados, seu pressuposto é que se CSI for igual a 1 (um), significa que a série de dados que foram detectadas pelo satélite são as mesmas observadas pelos pluviômetros e ao mesmo tempo não produzir nenhum alarme falso.

$$
\begin{gathered}
P O D=\frac{H}{H+F} \\
F A R=\frac{F}{H+F} \\
C S I=\frac{H}{H+M+F}
\end{gathered}
$$

Em que, H é a precipitação medida pelo pluviômetro e detectada pelo satélite; M é a precipitação observada pelo pluviômetro, mas não detectada; F é a precipitação detectada pelo satélite, mas não observada pelo pluviômetro. 


\section{RESULTADOS E DISCUSSÕES}

A Figura 2 apresenta a precipitação acumulada mensal nos postos pluviométricos instalados em Praia e Trindade; e a precipitação estimada pelo produto TRMM no período de 1998 - 2002, sobre a cidade de Praia capital de Cabo Verde. A precipitação pluviométrica apresentou sazonalidade característica com precipitações máximas durante agosto a outubro e menores durante dezembro a junho. Os meses mais secos, de julho e novembro são considerados de transição. De acordo com Lima (2012), o regime hidrológico na região está ligado a influência da Zona de Convergência Intertropical que atua no arquipélago e em toda África Ocidental enquanto que na época da seca o arquipélago está sobre influência dos ventos alísios.

As precipitações acumuladas anuais não ultrapassaram os 300 mm/ano, tanto nos postos pluviométricos quanto nas precipitações estimadas pelo produto TRMM. Isso se explica pelo fato da ilha de Santiago estar localizada no conjunto de ilhas a Sotavento; ilhas que sofrem grande influência dos ventos Sahelianos vindo do interior do continente africano, que impedem a formação de chuvas.

Os baixos valores de precipitação em Praia, estiveram associados a localização da ilha a Sotavento, local que sofre influência dos ventos Sahelianos vindos do interior do continente Africano, impedindo a formação de precipitação (SANCHEZ-MORENO et al., 2014).

Além disso, os aerossóis influenciam processos de superfície e troposférico pelos efeitos diretos, indiretos e semi-indiretos, acrescentando incerteza para compreensões climáticas antropogênicas. Os efeitos diretos são dominantes durante a temporada de inverno no hemisfério Norte com visibilidade reduzida em Cabo Verde quando se compara com a temporada de verão em que os efeitos indiretos podem influenciar significativamente a precipitação na região do Sahel (JENKIS et al., 2008).

A precipitação acumulada maior em agosto a outubro, não ultrapassou $180 \mathrm{~mm} / \mathrm{mês}$, período das chuvas no arquipélago de Cabo Verde em que está associado a monção tropical, que é controlada pelas migrações sazonais da Zona de Convergência Intertropical (ZCIT) em todo o Equador (SANCHEZMORENO et al., 2014).

Sanchez-Moreno (2014) ao analisarem os padrões de precipitação em Cabo Verde entre os anos de 1995 a 2010, observaram que houve interação entre a topografia, isso porque a formação da precipitação nas ilhas é complexa e variável, e sendo observadas nas medições em diferentes pontos. Por exemplo, precipitações de baixa intensidade moderada na baixa ilha de Santiago pode ter origem orográfica, enquanto, precipitações intensas (altas intensidades) podem ser convectivas ou até mesmo, ter formação frontal. 

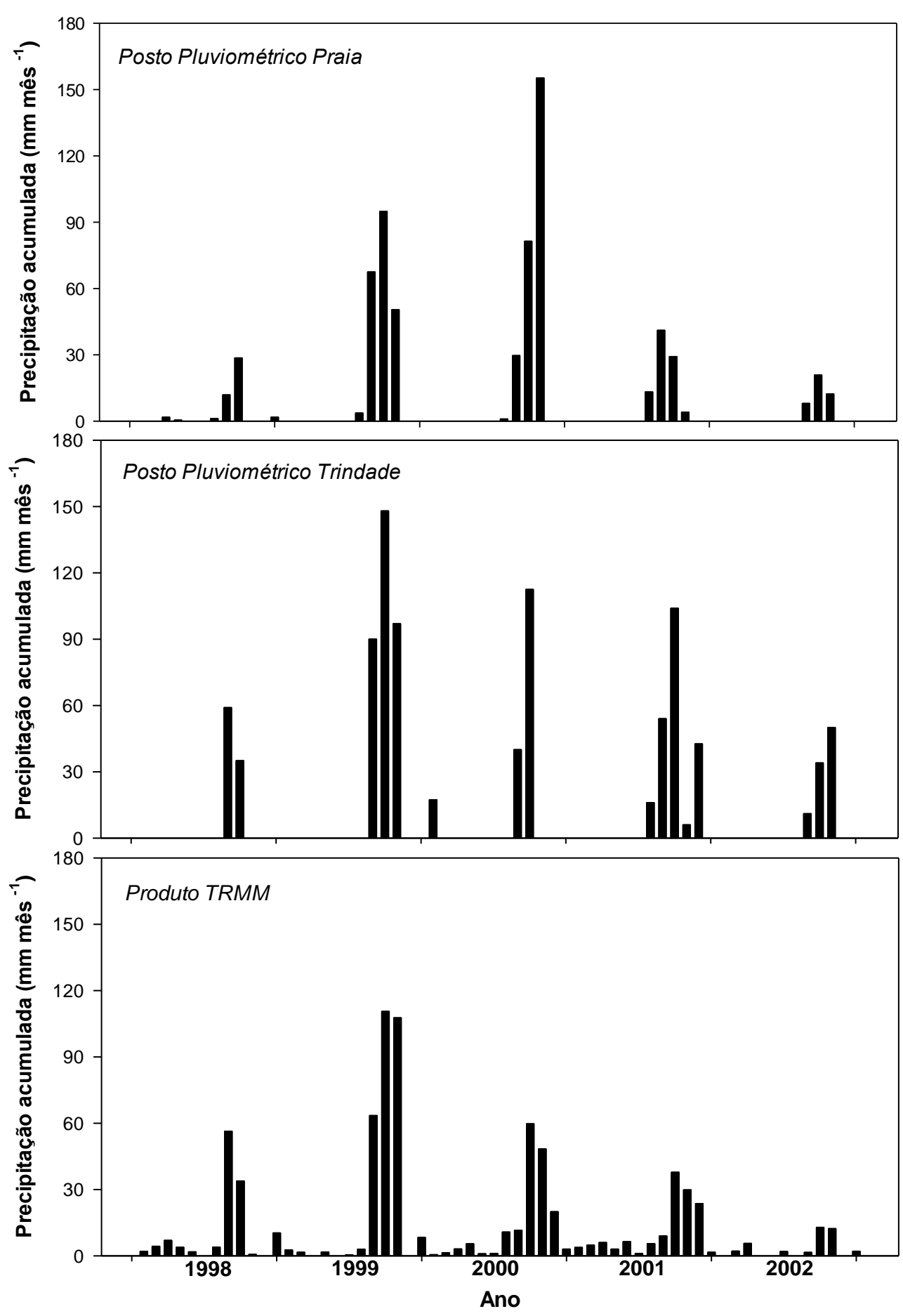

Figura 2 - Precipitação acumulada mensal nos postos pluviométricos de Praia, Trindade e a precipitação estimada pelo produto 3B43 TRMM no período de 1998 - 2002, sobre a cidade de Praia capital de Cabo Verde. 
Comparando os dados de pluviômetros e os valores de precipitação do 3B43 TRMM, observa-se uma boa coincidência entre os meses de chuva, ou seja, a sazonalidade da precipitação para os dois casos é semelhante. Nos postos pluviométricos os valores de precipitação foram registrados apenas nos meses de maior ocorrência (agosto, setembro e outubro) e nos outros meses os valores foram registrados com zero, significando que não choveu nesse período.

O produto 3B43 TRMM registrou valores significativos para os meses de maior ocorrência de precipitação, no entanto nos meses em que os postos pluviométricos registraram como zero o satélite estimou determinados valores ainda que bem inferior aos meses mais chuvosos, mas que tiveram uma parcela significativa para serem identificados pelo satélite.

Sanchez-Moreno et al. (2014) mencionaram que as estimativas de precipitação pelo produto TRMM detectam a tendência geral da série de dados e a direção das nuvens de chuva, ou seja, elas podem ter ocorrido nesse período, porém a precipitação não foi intensa o suficiente para atingir a superfície e, consequentemente, não sendo registradas pelos pluviômetros instalados na superfície.

Considerando a precipitação nos dois postos pluviométricos, as maiores precipitações ocorreram em Praia, indicando a variabilidade da precipitação em superfície, pois os postos estavam instalados na mesma cidade a alguns $\mathrm{km}$ de distância.

Isso pode indicar que as chuvas na região foram na maioria dos casos localizadas, e podem ser influenciadas pela altitude, pois o posto pluviométrico Trindade está localizado a $204 \mathrm{~m}$ de altitude enquanto que Praia à $64 \mathrm{~m}$. sendo essa a justificativa de se analisar os dados com os postos pluviométricos isolados com os dados estimados e posteriormente analisar os dados fazendo uma média entre os valores observados para compara-los com os dados estimados.

A Tabela 1 apresenta os resultados da análise estatística da série temporal anual e total entre os dados observados pelos postos pluviométricos e os dados estimados pelo produto 3B43 TRMM, assim como a média dos dados observados pelos postos pluviométricos com os dados estimados pelo produto.

$O$ índice de correlação linear ( $r$ ) realizado com as médias das precipitações variaram entre 0,66 e 0,96 indicando correlação satisfatória entre os dados estimados e os dados observados na maior parte dos anos. No entanto, a correlação linear com a serie temporal total apresentou $r$ de 0,74 em Praia de 0,85 em Trindade, considerando a média da precipitação nos postos pluviométricos, o índice foi 0,88 , indicando que ao fazer a diluição dos dados, a correlação entre os dados observados e os dados estimados melhora consideravelmente.

A raiz do erro médio quadrático apresentou menores valores considerando a relação entre a precipitação observada pelos postos pluviométricos e o produto 3B43 TRMM. No geral, a melhor relação foi observada entre a precipitação estimada pelo produto quando considerando a média da precipitação observada pelos postos pluviométricos na escala temporal total (1998-2002), aumentando a acurácia do produto com relação ao que foi observado em superfície. 
O TRMM apresentou tendência em superestimar a precipitação quando ocorreram menores índices pluviométricos (valores positivos) e subestimar em maiores índices pluviométricos (valores negativos), corroborando com Duan e Bastiaanssen (2013) na bacia do lago Tana, com Zheng e Zhu (2014) na China e Darand et al (2017) sobre o Irã.

Considerando a série temporal total (1998 a 2002), a relação entre os dados de superfície dos postos pluviométricos e o produto TRMM apresentaram erros relativos baixos, no entanto, como os índices pluviométricos da região foram relativamente baixos comparados a outras regiões tropicais, os mesmos podem não ser significativos ao ponto de não aceitar a validação das estimativas que o TRMM faz para essas regiões em que quase não ocorre precipitação ao longo dos anos.

Tabela 1 - Índices de Coeficiente de Correlação de Pearson ( $r$ ), Raiz Quadrada do Erro Médio Quadrático (RMSE), Erro Relativo (Errv (\%)), Probabilidade de Detecção (POD), Falso Alarme (FAR) e Índice de Sucesso (CSI) na Validação Por Pixel do Produto 3B43 V7 TRMM.

\begin{tabular}{|c|c|c|c|c|c|c|c|}
\hline \multirow{2}{*}{$\begin{array}{c}\text { Postos } \\
\text { Pluviométricos }\end{array}$} & \multirow[b]{2}{*}{ Índices } & \multicolumn{6}{|c|}{ Ano } \\
\hline & & 1998 & 1999 & 2000 & 2001 & 2002 & $\begin{array}{c}1998- \\
2002\end{array}$ \\
\hline \multirow{6}{*}{$\begin{array}{c}\text { Praia } \\
\text { versus } \\
\text { 3B43TRMM }\end{array}$} & $r$ & 0,75 & 0,94 & 0,86 & 0,38 & 0,86 & \multirow{3}{*}{$\begin{array}{c}0,74 \\
18,34 \\
0,15\end{array}$} \\
\hline & RMSE & 13,36 & 17,37 & 32,62 & 14,46 & 3,54 & \\
\hline & $\begin{array}{l}\text { Errv } \\
(\%)\end{array}$ & 1,69 & 0,38 & $-0,38$ & 0,51 & $-0,07$ & \\
\hline & POD & 1 & 1 & 1 & 1 & 1 & 1 \\
\hline & FAR & 0,40 & 0,60 & 0,58 & 0,67 & 0,67 & 0,58 \\
\hline & CSI & 0,60 & 0,40 & 0,42 & 0,33 & 0,33 & 0,42 \\
\hline \multirow[b]{3}{*}{$\begin{array}{c}\text { Trindade } \\
\text { versus }\end{array}$} & \multirow{2}{*}{$\begin{array}{c}r \\
\text { RMSE }\end{array}$} & 0,98 & 0,97 & 0,67 & 0,73 & 0,90 & \multirow{2}{*}{$\begin{array}{r}0,85 \\
17,32\end{array}$} \\
\hline & & 4,25 & 13,88 & 23,77 & 25,15 & 12,94 & \\
\hline & $\begin{array}{l}\text { Errv } \\
(\%)\end{array}$ & 0,31 & $-0,11$ & $-0,03$ & $-0,41$ & $-0,59$ & $-0,17$ \\
\hline \multirow[t]{4}{*}{ 3B43TRMM } & POD & 1 & 1 & 1 & 1 & 1 & \multirow{3}{*}{$\begin{array}{c}1 \\
0,70 \\
0,30 \\
\end{array}$} \\
\hline & FAR & 0,80 & 0,70 & 0,75 & 0,58 & 0,67 & \\
\hline & CSI & 0,33 & 0,33 & 0,30 & 0,38 & 0,33 & \\
\hline & $r$ & 0,96 & 0,96 & 0,94 & 0,66 & 0,91 & \multirow{2}{*}{$\begin{array}{c}0,89 \\
12,24\end{array}$} \\
\hline \multirow{5}{*}{$\begin{array}{l}\text { Média dos } \\
\text { postos } \\
\text { versus } \\
\text { 3B43TRMM }\end{array}$} & RMSE & 7,15 & 11,50 & 16,86 & 16,21 & 7,52 & \\
\hline & $\begin{array}{l}\text { Errv } \\
(\%)\end{array}$ & 0,77 & 0,08 & $-0,24$ & $-0,15$ & $-0,44$ & $-0,04$ \\
\hline & POD & 1 & 1 & 1 & 1 & 1 & 1 \\
\hline & FAR & 0,40 & 0,60 & 0,50 & 0,58 & 0,67 & 0,55 \\
\hline & CSI & 0,60 & 0,40 & 0,50 & 0,42 & 0,33 & 0,45 \\
\hline
\end{tabular}

Os Índices de Probabilidade de Detecção os valores foram satisfatórios (Tabela 1), indicando que o satélite detectou toda precipitação que ocorreu em superfície, no entanto, o índice Alarme Falso demonstrou que o produto de estimativa de precipitação pelo TRMM detecta falsamente o que foi observado em superfície aproximadamente $60 \%$ dos casos. 
Darand et al. (2017) explicam que o baixo desempenho em regiões com índices pluviométricos baixos, provavelmente pela adoção de algoritmos de estimativa de precipitação por satélite para capturar a precipitação em regiões quentes, que geralmente ocorrem em períodos com altos índices de precipitações e excluir a chuva de nuvens cirrosas finas, que geralmente são muito frias e de ocorrências no período de seca.

O Índice de Sucesso indicou quais foram as situações em que o satélite estimou corretamente os eventos de precipitação que foram observados em superfície. No geral, os melhores índices foram entre a média dos postos pluviométricos e o produto 3B43 TRMM considerando a série temporal total (1998 a 2002).

É importante ressaltar que apesar das estimativas terem uma boa probabilidade de detecção não significa que a estimativa será feita integralmente de acordo com as observações feitas em superfície. Portanto, esses índices não podem ser avaliados separadamente, pois um é complemento do outro, de maneira que na medida que o primeiro índice indica tudo o que foi estimado em razão do observado o outro mostra o que foi detectado de forma correta e incorretamente.

Entretanto, não se pode afirmar com exatidão que o produto de estimativa de precipitação não apresenta dados satisfatórios, deve-se levar em consideração as escalas de cada dado. Enquanto, o produto estima a precipitação sobre uma área total de um pixel, as observações feitas em superfície são pontuais, existindo incompatibilidade de escala de dados (escala de pontos versus escala de pixels de $0,25^{\circ}$ ) (DUAN e BASTIAANSSEN, 2013).

\section{CONCLUSÕES}

Por meio dos resultados obtidos é possível concluir que: apesar de na maioria dos casos as estimativas estarem de acordo com os dados observados, houve casos em que essa relação não foi satisfatória, seria o caso de talvez estipular limites para os valores de precipitação para que os resultados se comportassem um pouco mais significativos, porém é necessário ressaltar que na medida que passou-se a avaliar os dados para serie temporal os resultados foram mais satisfatórios.

Além disso, a precipitação estimada pelo produto TRMM pode ser utilizada como forma alternativa para a obtenção da precipitação em regiões com escassez de postos pluviométricos ou em casos em que haja falhas de dados em series temporais e espaciais, e aliados a estatística contribui para a prevenção de desastres naturais e climatológicos referentes a precipitação.

\section{REFERÊNCIAS}

COLLISCHONN, B. Uso de precipitação estimada pelo satélite TRMM em modelo hidrológico distribuído. Dissertação de mestrado, IPH-UFRGS, Porto Alegre - RS, 2006.

COSTA, F. L. Processos erosivos actuais na bacia da Ribeira Seca (Santiago Oriental - Cabo Verde). Garcia de Orta: Revista do Instituto de Investigação Científica Tropical, v. 15, n. 1, p. 29-34, 1996. 
DARAND, M; Amanollahi, J.; Zandkarimi, S. Evaluation of the performance of TRMM Multi-satellite Precipitation Analysis (TMPA) estimation over Iran. Atmospheric Research, n. 190, p. 121-127, 2017.

HIROSE, M.; TAKAYABU Y. N.; HAMADA, A. SHIGE, S.; YAMAMOTO, M. K. Impact of long-term observation on the sampling characteristics of TRMM PR precipitation. Journal of Applied Meteorology and Climatology, v. 56, n. 3, p. 713-723, 2017.

HOU, A. Y.; KAKAR, R. K.; NEECK, S.; AZARBARZIN, A. A.; KUMMEROW, C. D.; KOJIMA, M.; OKI, R.; NAKAMURA, K.; IGUCHI, T. The Global Precipitation Measurement Mission. Bulletin of the American Meteorological Society, v. 95, $p$ 701-722, 2014.

HUFFMAN, G. J.; ADLER, R. F.; BOLVIN, D. T.; GU, G.; NELKIN, E. J.; BOWMAN, K. P.; HONG, Y.; STOCKER, E. F.; WOLFF, D. B. The TRMM Multisatellite Precipitation Analysis (TMPA): quasi-global, multiyear, combined-sensor precipitation estimates at fine scales. Journal of Hydrometeorology, v.8, n. 1, p. 38-55, 2007.

HUFFMAN, G. J.; BOLVIN, D. T.; NELKIN, E. J. TRMM and Other Data Precipitation Data Set Documentation, Mesoscale Atmospheric Processes Laboratory. NASA Global Chang Master. Directory Doc., p 44. Disponível em: <http://pmm.nasa.gov/sites/default/files/document_files/3B42_3B43_doc_V7.p df $>$.

ISLAM, M. N.; UYEDA, $H$. Use of TRMM in determining the climatic characteristics of rainfall over Bangladesh. Remote Sensing of Environment, $v$. 108, n. 3, p. 264-276, 2007.

JENKINS, G. S.; PRATT, A. S.; HEYMSFIELD, A. Possible linkages between Saharan dust and tropical cyclone rain band invigoration in eastern Atlantic during NAMMA-06. Geophysical Research Letters, v. 35, L08815, doi: 10.1029/2008GL034072, 2008.

KIDD, C.; HUFFMAN, G. J. Global precipitation measurement. Meteorological Applications, v 18, p. 334-353. 2011.

KITTEL, C. M. M.; NIELSEN, K.; TØTTRUP, C.; BAUER-GOTTWEIN, P. Informing a hydrological model of the Ogooué with multi-mission remote sensing data. In: EGU General Assembly Conference Abstracts, p. 8182, 2017.

KUMMEROW, C. D.; BARNES, W., KOZU, T.; SHIUE, J.; SIMPSON, J. The Tropical Rainfall Measuring Mission (TRMM) sensor package. Journal of Atmospheric and Oceanic Technology, v. 15, p 809-817, 1998.

KUMMEROW, C.; HONG, Y.; OLSON, W. S.; YANG, S.; ADLER, R. F.; MCCOLLUM, J.; FERRARO, R.; PETTY, G.; WILHEIT, T. T. The evolution of the Goddard Profiling Algorithm (GPROF) for rainfall estimation from passive microwave sensors. Journal of Applied Meteorology, v.40, 1801-1820, 2001.

LIMA, I. P. Inundações urbanas: desafios ao ordenamento do território. O caso da cidade da Praia (Cabo Verde). Dissertação de mestrado. Porto Alegre - RS, 2012.

MEHTA, A. V.; YANG, S. Precipitation climatology over Mediterranean Basin from ten years of TRMM measurements. Advances Geosciences, v. 17, p 87-91, 2008. 
NASTOS, P.T.; KAPSOMENAKIS, J.; PHILANDRAS, K.M. Evaluation of the TRMM 3B43 gridded precipitation estimates over Greece. Atmospheric Research, v. 169 , p. 497-514, 2016.

SANCHEZ-MORENO, J. F.; MANNAERTS, C.; JETTEN, V. Applicability of satellite rainfall estimates for erosion studies in small offshore areas: a case study in Cape Verde Islands. Catena, v. 121, p. 365-374, 2014.

SANCHEZ-MORENO, J. F.; MANNAERTS, C.; JETTEN, V. Influence of topography on rainfall variability in Santiago Island, Cape Verde. International Journal of Climatology, n. 34, p. 1081-1097, 2014.

SILVA, D. F.; PANTANO, A. P.; CAMARGO, M. B. P. Analise de dados de precipitação estimados pelo satélite TRMM para o Vale do médio Parapanema SP. Reveng. Engenharia na agricultura, Viçosa - MG, v.21, n. 2, p. 138-147, 2013.

YANG, S.; SMITH, E. A. Convective - stratiform precipitation variability at seasonal scale from eight years of TRMM observations: implications for multiple modes of diurnal variability. Journal of Climate, v. 21, p. 4087-4114, 2008 